\title{
Correlations between capnographic waveforms and peak flow meter measurement in emergency department management of asthma
}

\author{
N. A. R. Nik Hisamuddin • A. Rashidi • K. S. Chew • \\ J. Kamaruddin • Z. Idzwan • A. H. Teo
}

Received: 13 May 2008 / Accepted: 9 January 2009 / Published online: 24 February 2009

(C) Springer-Verlag London Ltd 2009

\begin{abstract}
Background The usual method for initial assessment of an acute asthma attack in the emergency room includes the use of peak flow measurement and clinical parameters. Both methods have their own disadvantages such as poor cooperation/effort from patients (peak flow meter) and lack of objective assessment (clinical parameters). We were looking into other methods for the initial asthma assessment, namely the use of capnography. The normal capnogram has an almost square wave pattern comprising phase 1, slope phase 2, plateau phase 3, phase 4 and angle $\alpha$ (between slopes 2 and 3). The changes in asthma include decrease in slope of phase 2, increase in slope 3 and opening of angle $\alpha$.

Aims Our objective was to compare and assess the correlation between the changes in capnographic indices
\end{abstract}

The views expressed in this paper are those of the author(s) and not those of the editors, editorial board or publisher.

N. A. R. Nik Hisamuddin $(\bowtie) \cdot$ A. Rashidi $\cdot$ K. S. Chew

Department of Emergency Medicine, Health Campus,

Hospital Universiti Sains Malaysia,

Kubang Kerian, Kelantan, Malaysia

e-mail: nhliza@hotmail.com

\section{J. Kamaruddin}

Intensivist Care, Health Campus,

Hospital Universiti Sains Malaysia,

Kubang Kerian, Kelantan, Malaysia

\section{Z. Idzwan}

Department of Emergency Medicine,

Universiti Malaya Medical Center,

Kuala Lumpur, Malaysia

A. H. Teo

Emergency Department, Penang Hospital,

Penang, Malaysia and peak flow measurement in non-intubated acute asthmatic patients attending the emergency room.

Methods We carried out a prospective study in a university hospital emergency department (ED). One hundred and twenty eight patients with acute asthma were monitored with peak flow measurements and then had a nasal cannula attached for microstream sampling of expired carbon dioxide. The capnographic waveform was recorded onto a PC card for indices analysis. The patients were treated according to departmental protocols. After treatment, when they were adjudged well for discharge, a second set of results was obtained for peak flow measurements and capnographic waveform recording. The pre-treatment and post-treatment results were then compared with paired samples $t$-test analysis. Simple and canonical correlations were performed to determine correlations between the assessment methods. A $p$ value of below 0.05 was taken to be significant.

Results Peak flow measurements showed significant improvements post-treatment $(p<0.001)$. On the capnographic waveform, there was a significant difference in the slope of phase $3(p<0.001)$ and alpha angle $(p<0.001)$, but not in phase 2 slope $(p=0.35)$. Correlation studies done between the assessment methods and indices readings did not show strong correlations either between the measurements or the magnitude of change pre-treatment and post-treatment.

Conclusion Peak flow measurements and capnographic waveform indices can indicate improvements in airway diameter in acute asthmatics in the ED. Even though the two assessment methods did not correlate statistically, capnographic waveform analysis presents several advantages in that it is effort independent and provides continuous monitoring of normal tidal respiration. They can be proposed for the monitoring of asthmatics in the ED. 
Keywords Asthma Capnography.

Peak flow measurement $\cdot$ Emergency room

\section{Introduction}

Capnometry, discrete measurements of carbon dioxide concentrations, was first developed during the Second World War as a means of monitoring the internal environment of submarines [1]. In the 1950s, capnometers were used experimentally during anaesthesia to measure expired $\mathrm{CO}_{2}$. But it was only in the early 1980s that capnometry became widely used mainly in anaesthetic practice [2]. Today, capnography is considered to be essential in monitoring metabolic and respiratory functions during anaesthesia. Its role has spread beyond the realms of anaesthesia and capnography is now used in emergency medicine to confirm and verify endotracheal tube placement, monitor ventilatory status of patients with impaired respiratory function, monitor ventilation of patients during sedation/analgesia, evaluate ventilator settings and circuit integrity, assess effectiveness of cardiopulmonary resuscitation, and for early detection of changes in airway resistance and circulatory collapse. The normal capnogram has an almost square wave pattern (Fig. 1), marked by alternating inspiratory $\left(\mathrm{P}_{\text {insp }} \mathrm{CO}_{2}\right.$ equals zero) and expiratory phases $[3,4]$. Expiration itself consists of three successive phases: (1) a latency phase (phase 1), corresponding to the expiration of the anatomical dead space $\left(\mathrm{P}_{\exp } \mathrm{CO}_{2}\right.$ equals zero), which is indistinguishable from the preceding inspiration; (2) slope phase (phase 2) marked by a very rapid rise in $\mathrm{P}_{\text {exp }} \mathrm{CO}_{2}$, corresponding to expiration of mixed air; and (3) plateau phase (phase 3), reflecting the elimination of alveolar air (slightly increasing $\mathrm{P}_{\exp } \mathrm{CO}_{2}$ ) resulting in a peak at the end of tidal expiration $\left(\mathrm{P}_{\mathrm{et}} \mathrm{CO}_{2}\right.$ close to alveolar carbon dioxide tensions $\mathrm{P}_{\mathrm{A}} \mathrm{CO}_{2}$ ) [5, 6]. The end-tidal peak is occasionally referred to as the end-tidal point. This well- defined shape of the normal capnograph depends on a variety of factors. Normal aerobic metabolism will consume oxygen and result in the production of carbon dioxide. This will be carried by an adequately functioning circulatory system to the lungs where, in the normal lung, matched gas distribution and alveolar ventilation with pulmonary perfusion will ensure normal gas exchange. The elimination of alveolar air is synchronous and this is reflected by the sudden rise of phase 2 and the subsequent elimination of alveolar air, which is indicated by the almost horizontal plateau of phase 3 .

Asthma is a disease characterized by bronchial hyperreactivity, inflammatory exudates and mucous plugging. The hallmark of asthma is the narrowing of the smaller airways causing obstruction to flow within the airways especially during expiration. In asthma, airway obstruction causes regional decreases in airflow and consequently, alveolar ventilation. This is responsible for the "parallel heterogeneity" of ventilation-perfusion ratios (V/Q ratios). On the capnogram, this causes deformation of the normal curve, marked by the loss of verticality of phase 2 , opening of the angle between phases 2 and 3 (alpha angle also known as angle Q) and the increased inclination of phase 3 . In severe cases, the capnogram takes on a "shark fin" appearance (Fig. 2). These changes in the capnogram are of particular interest as they indicate changes in airflow.

Asthmatics who present to the emergency department (ED) with acute attacks of asthma are often required to perform forced expiration manoeuvres to assess the degree of airway obstruction and thereby the severity of the attack. These manoeuvres are then repeated post-treatment to confirm the return to baseline status. These manoeuvres have been well studied and documented to accurately assess severity of bronchospasm and they form the basis of almost all monitoring of asthmatics in EDs throughout the world. Nowak et al. in a series of landmark studies conducted in the late 1970 s and early 1980 s reported on spirometric criteria in patients with acute asthma in the ED [7]. These
Fig. 1 A normal single capnographic wave

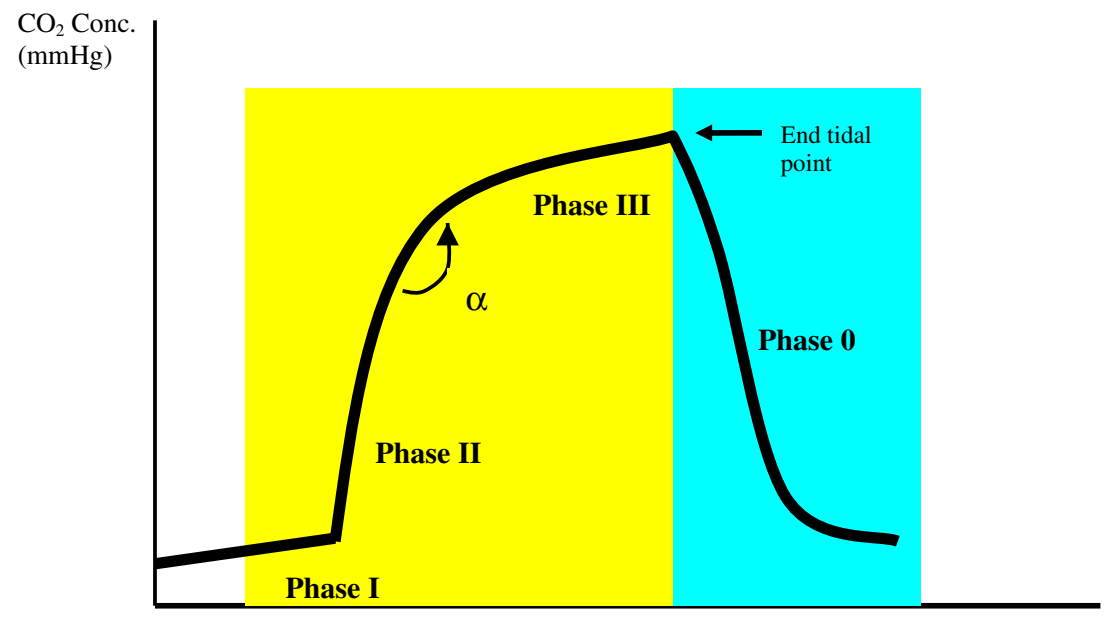

Time (s) 
Fig. 2 The capnographic waveform - changes in asthma

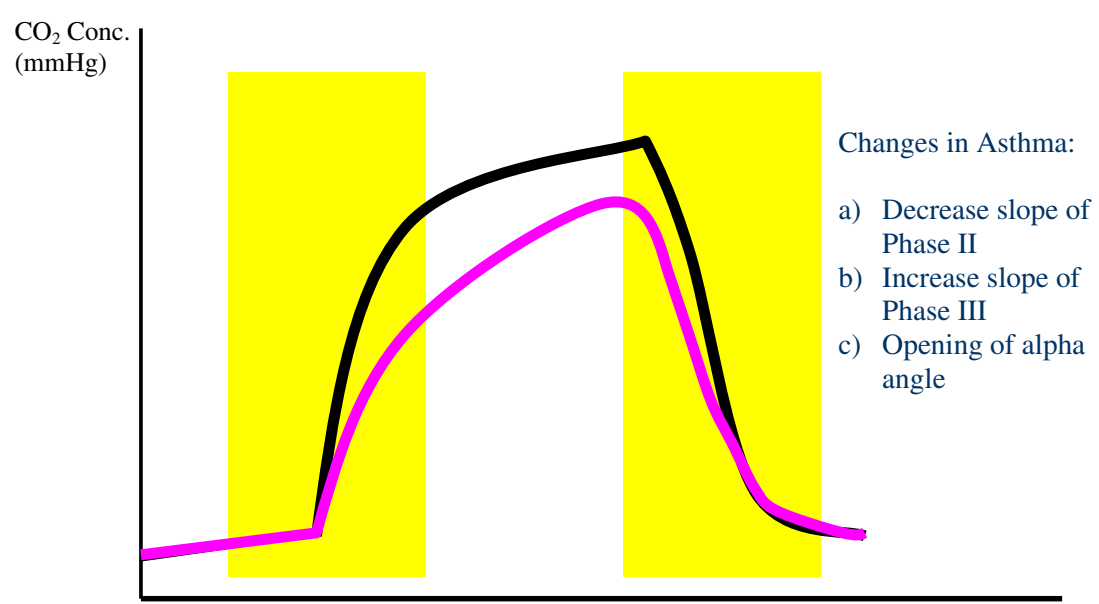

Time (s) studies were among the first to introduce spirometric criteria for admission and discharge of patients from the ED. The data from these studies formed the basis of many of the recommendations concerning peak expiratory flow rate (PEFR) values that are needed to discharge patients from the ED that are in use today [8]. However, there are several limitations to forced expiration manoeuvres. Most significantly, they require an active and technically correct effort to perform such manoeuvres [9]. Often, spirometric measurements depend on whether medical staffs are able to explain, motivate and guide patients to perform the manoeuvres properly and whether patients are willing and able to do them [10]. Very often, this difficulty precludes the use of these measurements in children, the elderly and the very dyspnoeic patient. Capnography offers several advantages in this aspect; it is non-invasive, non-effort related and independent of the patient's cooperation. It does not interfere with the administration of oxygen nor does it delay onset of nebulizer therapy. In fact, continuous monitoring of patients can be done during their nebulizer therapy and throughout their stay in the ED. It is this aspect of capnographic monitoring that greatly interests many clinicians and researchers alike.

We conducted a study on patients who presented with acute exacerbation of asthma to the emergency room in the Hospital Universiti Sains Malaysia and carried out comparison and correlation studies between the peak flow measurement and the indices from the capnograph. The research was approved by the University Hospital Ethics and Technical Research Committee and was carried out as a partial fulfilment for a Masters Degree in Emergency Medicine.

\section{Methodology}

We conducted a prospective study on patients presenting to the ED with symptoms suggestive of asthma. This study was conducted in the ED of Hospital Universiti Sains
Malaysia in Kubang Kerian, Kelantan. It is a regional tertiary referral centre with an attendance rate exceeding 60,000 patients per year for the ED. It is also a teaching institution university hospital involved in the training of undergraduate medical students and residency-based specialty training in many fields including emergency medicine.

Inclusion criteria for enrolment into this study were all adult patients who presented with symptoms of breathlessness and/or wheezing and a self-reported previous history of asthma. The Malaysian Constitution recognizes individuals with age of 12 years and above as adults and the same rule applies to the hospital admissions. The paediatric population was excluded for one obvious reason, that is the difficulty to obtain the PEFR reading. The diagnosis was further confirmed by looking into the patients' old hospital notes. Any patients with ambiguous diagnosis were excluded from the study. These patients were initially examined clinically by doctors working in the ED who assessed and documented the severity of the attack via spirometric and clinical parameters. The patients were managed according to standard protocol for acute exacerbation of asthma as recommended by the Malaysian Thoracic Society. These patients were then started on nebulizer therapy and enrolled in the study. Initial verbal consent was taken if patients were in a state of breathlessness or written consent was taken from the relatives if they were present during the emergency room admission. Patients were excluded from the study if they presented with severe life-threatening events where priority and attention was directed towards treatment. They were also excluded if chronic obstructive pulmonary disease (COPD) was suspected to be the primary diagnosis due to the irreversibility of at least some airway obstruction, as is well documented in this disease [11]. Inability to perform forced expiration manoeuvres, either pre-treatment or post-treatment, excluded any patient from enrolment. Anticipating this difficulty, we had decided to exclude children from the study. All patients whose 
symptoms were not completely relieved by nebulizer therapy and/or required admission were also excluded.

Patients were instructed to blow forcefully into the Wright's ${ }^{\circledR}$ Mini Peak Flow Meter (PEFR) thrice and all three measurements were recorded. For the purpose of analysis, the highest reading constituted the PEFR reading for the patient. The PEFR readings were represented as percentage of expected based on the normogram for prediction of PEFR based on age and height in men and women. Once the decision to start nebulizer therapy was given, the attending physician initiated capnographic monitoring in tandem with ongoing therapy. In this study we used a Novametrix Capnogard ${ }^{\circledR}$ capnograph, manufactured in the USA, which needed daily calibration. A nasal cannula, which served as the sampling port for sidestream capnography, was applied to the patient. They were then directed to breathe normally. One end of this specially designed nasal cannula provided oxygen from a humidified wall source (all patients in this study were given oxygen at a rate of $31 / \mathrm{min}$ via this nasal cannula) whereas the other end was connected to an aspiration pump and the sampling port $[12,13]$. The capnogram was then recorded and stored within a PC card. Waveforms were deemed adequate once at least three waveforms of regular morphology, without significant artefacts, were noted. Nebulizer therapy was then started with the nasal cannula in situ. All patients were treated with oxygen-driven wet nebulizers. At the conclusion of nebulizer therapy, once the attending doctor had decided that the patient was "fit for discharge", the post-treatment capnographic waveforms and PEFR were again recorded and stored [13]. Carbon dioxide readings were measured 48 times/s, i.e. at intervals of $0.02 \mathrm{~s}$. The capnogram recorded was stored in "comma-separated variable" (*.csv) data files for analysis. The capnograph machine was calibrated daily as recommended by the manufacturer.

Each capnograph was analysed for phase 2 slope (referred to subsequently as "slope") and phase 3 slope (referred to as "plateau"). The slope and plateau lines would create an obtuse angle with each other and this we referred to as angle $\alpha$. In all graphical analysis, the difficulty in producing computerized analysis always lies in the identification of constants or specifics within each waveform that allow for repeated and reproducible analysis. With this in mind, we decided to study the slope of phase 2 as measured for $0.25 \mathrm{~s}$ from the first point where the measured $\mathrm{CO}_{2}$ first rose above $4 \mathrm{mmHg}$ and the slope of phase 3 as measured for $0.75-0.25 \mathrm{~s}$ (total time $0.5 \mathrm{~s}$ ) from the end-tidal point. All patients' capnographs had three readings taken for slope and plateau, and therefore three values of $\alpha$ were calculated. Mean readings were then taken of the three initial readings. Variables were reported as mean with standard deviations. Statistical analysis was performed using SPSS (Version 9.0) and Stata (Version 7.0). Among the statistical tests used were frequency analysis, paired $t$-tests for comparison of means, simple and canonical correlations to determine correlations between tests and Wilcoxon non-parametric signed rank test for analysis of categorical data. While it must be recognized that blood pressures, respiratory rates and pulse rates are widely different from patient to patient and dependent on age, sex, underlying disease and degree of anxiety besides severity of disease, the paired $t$-test only compared changes in each individual patient thereby negating any interindividual variability. A value of $p<0.05$ was considered to be statistically significant.

\section{Results}

A total of 128 patients were initially enrolled in this study. Eight patients were not included in the study due to inability to analyse their capnographic records, primarily due to lack of adequate waveforms not deformed by artefacts. Sixteen patients were unable to perform the PEFR measurement due to poor effort and to distress from breathlessness and were hence excluded from the study. Four other patients were excluded due to missing or incomplete data. Data from 100 patients were submitted for analysis.

The age of our patients in this study ranged from 12 to 71 years with a mean of 35.2 years [ $95 \%$ confidence interval (CI): 22.5-45.3]. There were 56 male and 44 female patients. The range for height was from 137 to $176 \mathrm{~cm}$ with a mean of $158.7 \mathrm{~cm}$ (95\% CI: 45.3-172.8) whereas their weights ranged from 35 to $100 \mathrm{~kg}$ with a mean of $60.6 \mathrm{~kg}(\mathrm{SD} \pm 17.1 \mathrm{~kg})$. Of the 100 patients, $30 \%$ $(n=30)$ received just nebulized Salbutamol as their treatment in the ED; $53.3 \%(n=53)$ had nebulized Salbutamol followed by Salbutamol with Ipratropium, and the remaining $16.7 \%(n=17)$ had the above with I.V. bolus of Hydrocortisone $200 \mathrm{mg}$. Of the patients, $87 \%(n=87)$ were discharged from the emergency room with outpatient follow-up and none of them returned with the same complaint within the $24 \mathrm{~h}$ post-discharge; $13 \%(n=13)$ of them required further 24-h observation in the emergency room observation ward before being discharged home. None of the patients were admitted to the general medical ward (Table 1).

Initial PEFR readings were used to grade the severity of the asthmatic attack. Of the 100 patients in the study, $63 \%$ $(n=63)$ had a PEFR $<50 \%$ of expected on admission; $26 \%$ $(n=26)$ had an initial PEFR between 50 and $80 \%$ and $11 \%$ $(n=11)$ had a PEFR of more than $80 \%$ of expected. At the conclusion of therapy, when all patients were fit for discharge, 67 in total or $66.7 \%$ now had a PEFR of between 50 and $80 \%$ and $33 \%(n=33)$ had a PEFR of more than $80 \%$ of expected. Paired samples analysis showed a 
Table 1 Patient characteristics

Characteristic

Median age, years (range)

$35.2(12-71)$

Sex female:male (ratio)

$1: 1.5(40: 60)$

Median weight, kg (range)

$60.6(35-100)$

Height, cm (range)

$158.7(137-176)$

Patients received nebulized beta 2 -agonist alone

$30 \%(n=30)$

Patients received nebulized beta ${ }_{2}$-agonist and ipratropium bromide

$53 \%(n=53)$

Patients received intravenous hydrocortisone

$17 \%(n=17)$

Patients received combination beta $_{2}$-agonist and steroid inhaler on discharge

Patients discharged home after completing treatment in emergency room

$100 \%(n=100)$

Patients admitted to emergency room observation ward (maximum 24-h stay)

$87 \%(n=87)$

$13 \%(n=13)$

significant difference between pre- and post-PEFR values $(p<0.001)$ (Fig. 3). End-tidal carbon dioxide $\left(\mathrm{EtCO}_{2}\right)$ levels were also recorded pre- and post-treatment. $\mathrm{EtCO}_{2}$ levels pre-treatment ranged from 24 to $49 \mathrm{mmHg}$ with a mean of $36.8 \mathrm{mmHg}$ (95\% CI: 32.4-41.7). This was essentially unchanged post-treatment (range: $30-46 \mathrm{mmHg}$; mean: $36.7 \mathrm{mmHg}$; 95\% CI: 31.2-38.4). The paired $t$-test did not show any significant difference $(p=0.871)$.

Gradient values for phase 2 had a mean of $2.61(95 \%$ CI: 2.11-2.93) pre-treatment and a mean of $2.74(95 \%$ CI: $2.54-2.95)$ post-treatment. The paired $t$-test showed this to have no significant difference $(p=0.35)$. Gradient values for phase 3 had a mean of 0.44 (95\% CI: $0.33-0.47)$ pretreatment and a mean of 0.23 ( $95 \% \mathrm{CI}$ : $0.21-0.28)$ posttreatment. This reduction was markedly significant $(p<0.001)$. The angle $\alpha$ was calculated from the observed gradients of phases 2 and 3. The pre-treatment mean of $134.36(95 \%$ CI: 129.53-138.74) was significantly higher than the posttreatment mean of 123.27 (95\% CI: 121.25-127.96) $(p<0.001)$. Simple correlations were performed for numerical data whereas canonical correlations were used to analyse correlations between groups of variables. Correlation studies

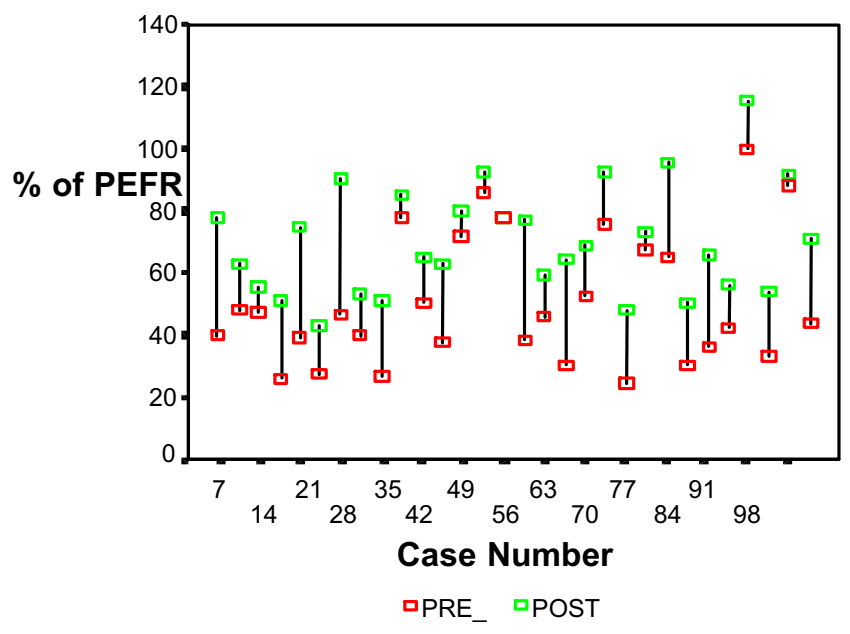

Fig. 3 Peak flow rates pre-treatment and post-treatment were conducted to attempt to correlate PEFR readings (analysed as percentage of expected) and graphical indices (phases 2 and 3 slopes and angle $\alpha$ ) in their ability to detect changes pre-treatment and post-treatment.

There were poor correlations between pre-treatment PEFR readings and graphical indices, i.e. between PEFR and phase 2 slope ( $r=0.60,95 \%$ CI: $0.55-0.68)$, and phase 3 slope $(r=-0.43,95 \%$ CI: -0.36 to -0.48$)$ and $\alpha(r=-0.57$, 95\% CI: -0.48 to -0.61$)$. Post-treatment, we also noted weak correlations between PEFR readings and graphical indices: phase $2(r=0.21,95 \% \mathrm{CI}: 0.17-0.28)$, phase 3 $(r=-0.39,95 \%$ CI: -0.31 to -0.45$)$ and $\alpha(r=-0.48,95 \%$ CI: -0.41 to -0.54 ). (Note: negative $r$ values indicate inverse/negative correlation). We also looked at the magnitude of change in PEFR and graphical indices pre- and posttreatment. We then conducted correlation studies after generating variables of differences. There was weak correlation between the magnitude of change in PEFR and phase 2 pre- and post-treatment $(r=0.48)$. Similarly, there were only weak negative correlations between the magnitude of change in PEFR and phase $3(r=-0.24,95 \%$ CI: -0.18 to $-0.31)$ and $\alpha(r=-0.31,95 \%$ CI: -0.26 to -0.38$)$ pre- and post-treatments. In conclusion, there were poor correlations between the magnitudes of change in PEFR and change in graphical indices, pre-treatment and post-treatment.

\section{Discussion}

Many of the previous studies on capnography in asthmatics have been conducted in more controlled situations, either with asthmatic patients in histamine challenge tests or whose asthma status has been stable for a few hours [14, 15]. On the contrary, this study was conducted on asthmatic patients presenting to the ED with an acute attack of asthma. We presume our findings are reflective of the situation in the ED, given our study population.

This study stands on one central pillar, that is the attending doctor according to departmental protocol (based 
on the Malaysian Thoracic Society guidelines) treats all patients in the study population without any influence from the researcher [16]. The researcher who was blinded to the clinical scenario analysed the data post hoc. Unfortunately it was hard to blind the attending physicians and the patients as the capnograph monitor was located next to the patients' beds. In other words, the ED doctor decided if the patient had an asthmatic attack and whether treatment was necessary. The end point of treatment, also decided by this doctor, occured when the patient was adjudged well and safe for discharge. We monitored these patients with clinical parameters, peak flow readings and capnographic waveform recordings. Capnographic waveform analysis is relatively new and is, as yet, unproven in its ability to detect changes in the airway of the asthmatic patient.

Currently, peak flow measurements are the most widely used method of objectively monitoring patients with acute asthma. Theoretically, peak flow rates are more a function of the diameter of the larger airways while forced expiratory volume in $1 \mathrm{~s}\left(\mathrm{FEV}_{1}\right)$ or $\mathrm{FEV}_{1} /$ forced vital capacity (FVC) reflect the smaller airways. $\mathrm{FEV}_{1}$ and $\mathrm{FEV}_{1} / \mathrm{FVC}$ are technically difficult to perform in the ED and involve cumbersome equipment. Many studies have shown that PEFR can be reliably used not only as a reflection of severity of the asthmatic attack, but also to indicate when patients should be admitted or can be discharged [17-19]. The major drawback is its dependency on effort. The patient's ability to perform these manoeuvres is vital. Often, in the ED, we find patients unable to perform these manoeuvres. Some are unmotivated to perform any manoeuvre in the midst of their discomfort, anxiety and breathlessness. Many, having performed such tests before, claim a worsening of symptoms especially cough after the manoeuvre. Some have such severe bronchospasm that they are totally unable to generate any significant flow at all. In the context of a study, all this introduces bias and skewed results. This was reflected in the study when $12 \%(n=16)$ of the patients were excluded because they were too distressed to blow into the peak flow meter. Practically speaking, the inability of the patients to produce pre-treatment PEFR now renders any post-treatment reading less useful; and any PEFR reading obtained may be spuriously low as a result of poor performance. PEFR then cannot be used alone to assess and monitor a patient in the ED. These limitations of peak flow manoeuvres are well known; but for the lack of a better monitoring technique, it is still the most reliable, and so the most widely used technique today.

The capnographic waveform shows changes in bronchospasm that reflect the heterogeneity of the expired air. This is seen as a decrease in the slope of phase 2 and increase of the slope of phase 3 and the resultant increase in the alpha angle (angle $\alpha$ ). While gross changes are visible to the naked eye, e.g. "shark fin appearance", more subtle changes require measurement of the slopes and angles. In this study, all the capnograms produced were trapezoid shaped which is a very common shape for those who present with mild to moderate asthma. The shark fin shaped capnogram would be most likely found in patients presenting with a very severe asthma attack who unfortunately were excluded from the study due to inability to use the peak flow meter. Since this is still a new concept, all studies to date have done this manually $[15,16]$. We have taken a slightly different approach. Keeping in view that any future application of this concept would require computerized analysis of the capnographic waveform, we set out by using indices that can be identified easily by computerized algorithm. For the slope of phase 2, we obtained readings for a period of $0.2 \mathrm{~s}$ from the point when the capnographic waveform crosses the carbon dioxide reading of $4 \mathrm{mmHg}$, assuming that the carbon dioxide has to have come from the lungs to reach a level of $4 \mathrm{mmHg}$. The second point identified was the end-tidal point, already a commonly identified point in all capnometers used today. We measured the slope of phase 3 by measuring for a period of $0.5 \mathrm{~s}$ from a point $0.25 \mathrm{~s}$ from the end-tidal point. These readings were then subjected to trend line analysis, resulting in the slope. From the two slopes, the angle of alpha $(\alpha)$ was calculated.

You et al., in their series of studies, have shown that slopes of phases 2 and 3 change demonstrably with improvements in airway diameter. They concluded that indices based on the later parts of the capnographic waveform are more sensitive and display a stronger correlation with spirometric indices [5]. In our study, we noted a similar trend, where phase 2 slopes did not show any significant change pre-treatment and post-treatment $(p=0.35)$. This is in contrast with the significant changes seen in phase $3(p<0.001)$ and alpha angle (angle $\alpha)(p<0.001)$.

Although phase 3 slope and alpha angle (angle $\alpha$ ) changes were significantly different pre- and post-treatment, the correlations with peak flow meter measurements were poor. In other terms, capnographic waveform indices were able to detect improvements in the patients' bronchospasm posttreatment, as could peak flow measurements, but the magnitude of improvement was poorly correlated between the two monitoring techniques. The poor correlation between the two parameters could be attributed to the poor initial PEFR effort by the patients because of the acute breathlessness state, thus contributing to the poor magnitude of changes in the PEFR reading. The question that needs to be asked then is: which then is the better monitoring parameter? There are insufficient studies at the moment to conclude this to any degree of certainty. Therefore, whether capnography is more accurate at detecting changes in asthma can only be answered by further studies. We were also 
unable to compare the graphical index changes between the groups of patients who were discharged home and admitted to the ward because all the patients included in the study were discharged from the emergency room eventually.

There are certain weaknesses to this study that we must acknowledge. Firstly, the study population was small. Distributions with respect to age or sex were not noted; however, there is no evidence to suggest that the shape of the capnogram is dependent on age or sex. A source of bias would be the selection of waveforms for analysis; waveforms were recorded when the morphology looked "similar" to the researcher and analysis was conducted on waveforms that were not excluded or deformed by artefacts. This is a selection bias. There are several possible approaches to this problem. Firstly, strict exclusion criteria may be imposed to sieve out waveforms with artefacts. This runs the risk of analysing waveforms that may not be indicative of the situation or having too few waveforms to analyse. Secondly, as Goldman and Landis have proposed, recognition of the different waveform morphologies, either through neural network algorithms or as clusters of morphologically similar waveforms, can be programmed [2022]. On the whole, this will reduce the influence of waveforms with artefacts or sub-optimal waveforms and this may then form the basis of waveform selection for analysis.

At the present moment, research into capnography waveform analysis is still evolving. Multiple avenues are being explored. Though we are still unclear of the future directions of this research, and how capnographic waveform analysis will be conducted in the future, the potential for its use practically demands further research into this field.

\section{Conclusion}

Even though the study did not show strong correlation between the measurements of the capnographic indices and peak flow meter, the former did show significant changes preand post-treatment. More research is required in future to prove whether capnography has a potential in future as a method for monitoring the acute asthma patient, in particular to assist physicians in deciding the effectiveness of the treatment.
4. You B, Peslin R, Duvivier C et al (1994) Expiratory capnography in asthma: evaluation of various shape indices. Eur Respir J 7:318-323

5. You B, Mayeux D, Rkiek B, Autran N, Dang Vu V, Grilliat JP (1992) La capnographie expiratoire dans l'asthme: perspectives d'utilisation comme methode de monitorage. Rev Mal Respir 9:547-552

6. Bhavani-Shankar K, Philip JH (2000) Defining segments and phases of a time capnogram. Anesth Analg 91(4):973-977

7. Nowak RM, Gordon KR, Wroblewski DA et al (1979) Spirometric evaluation of acute bronchial asthma. JACEP 8:9-12

8. Nowak RM, Pensier MI, Sarkar DD et al (1982) Comparison of peak expiratory flow rate and FEV1 admission criteria for acute bronchial asthma. Ann Emerg Med 11(2):64-69

9. Hyatt RE (1986) Forced expiration. In: Fishman AP (ed) Handbook of physiology. American Physiological Society, Bethesda, pp 295-314

10. Primatesta P, Bost L, Dong W (1996) Health survery for England. Stationery Office, London

11. Fletcher CM (1976) The natural history of chronic bronchitis and emphysema. Oxford University Press, Oxford

12. Bowe EA, Boysen PG, Broome JA, Klein EF Jr (1989) Accurate determination of end-tidal carbon dioxide during administration of oxygen by nasal cannulae. J Clin Monit 5:105-110

13. Loughnan TE, Monagle J, Copland JM, Ranjan P, Chen MF (2000) A comparison of carbon dioxide monitoring and oxygenation between facemask and divided nasal cannula. Anaesth Intensive Care 28(2):151-154

14. Yaron M, Padyk P, Hutsinpiller M, Cairns CB (1996) Utility of expiratory capnogram in the assessment of bronchospasm. Ann Emerg Med 28:403-407

15. Egleston CV, Ben Aslam BH, Lambert MA (1997) Capnography for monitoring non-intubated spontaneously breathing patients in an emergency room setting. J Accid Emerg Med 14:222-224

16. Zainudin BMZ (1996) Guidelines on management of adult asthmaa consensus statement. Malaysian Thoracic Society

17. Fischl MA, Pitchenik A, Gardner LB (1981) An index predicting relapse and need for hospitalization in patients with acute bronchial asthma. N Engl J Med 305:783-789

18. Nowak RM, Pensler MI, Sarkar DD et al (1982) Comparison of peak expiratory flow rate and FEV1 admission criteria for acute bronchial asthma. Ann Emerg Med 11(2):64-69

19. Corre KA, Rothstein RJ (1985) Assessing severity of adult asthma and need for hospitalization. Ann Emerg Med 14:45-52

20. Landis B, Romano PM (1998) A scoring system for capnogram biofeedback: preliminary findings. Appl Psychophysiol Biofeedback 23(2):75-91

21. Goldman JM (2000) Development of clinical capnography analysis system. Director of Anaesthesiology Research, University of Colorado Health Sciences Center. Web-based paper http://www. acmeanesthesia.com/

22. Bhavani-Shankar K, Kumar AY, Moseley HS, Ahyee-Hallsworth R (1995) Terminology and the current limitations of time capnography: a brief review. J Clin Monit 11:175-182

\section{Conflicts of interest None.}

\section{References}

1. O'Flaherty D (1994) Capnography: principles and practice. BMJ Publishing Group, London

2. Smalhout B, Kalenda Z (1981) An atlas of capnography. Kerchebosch-Zeist, Amsterdam

3. Berrengo A, Cutilloa A (1961) Single-breath analysis of carbon dioxide concentration records. J Appl Physiol 16:522-530
Nik Ab. Rahman Nik Hisamuddin is a Senior Lecturer and Consultant in Emergency Medicine, Traumatology and Hyperbaric Medicine and Head of the Department of Emergency Medicine, Health Campus, Hospital Universiti Sains Malaysia, Kubang Kerian, Kelantan, Malaysia. He has qualified as a Clinical Fellow in Accident and Emergency at the Royal Infirmary of Edinburgh in Scotland and as a Hyperbaric Medicine and Diving Medical Officer at Universiti Sains Malaysia, Kubang Kerian, Kelantan, Malaysia. He has studied at Hyperbaric International in Key Largo, FL, at the Nix Medical Center for Hyperbaric and Wound Care, San Antonio, TX, at the University of Glasgow, Glasgow, Scotland and at the Warwick School, Warwick, UK. 